Acta Crystallographica Section F

Structural Biology

and Crystallization

Communications

ISSN 1744-3091

Emma Byres, David M. A. Martin and William N. Hunter*

Division of Biological Chemistry and Molecular Microbiology, School of Life Sciences, University of Dundee, Dundee DD1 5EH, Scotland

Correspondence e-mail: w.n.hunter@dundee.ac.uk

Received 19 April 2005

Accepted 9 May 2005

Online 1 June 2005

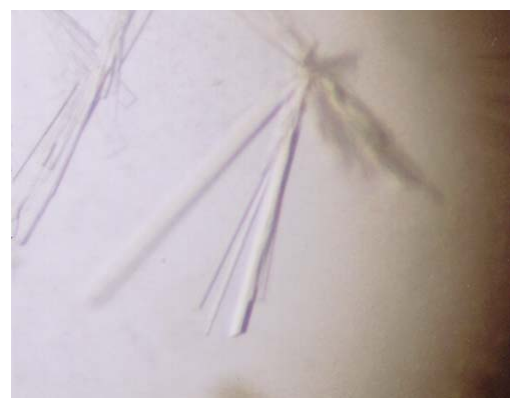

(C) 2005 International Union of Crystallography All rights reserved

\section{A preliminary crystallographic analysis of the putative mevalonate diphosphate decarboxylase from Trypanosoma brucei}

Mevalonate diphosphate decarboxylase catalyses the last and least well characterized step in the mevalonate pathway for the biosynthesis of isopentenyl pyrophosphate, an isoprenoid precursor. A gene predicted to encode the enzyme from Trypanosoma brucei has been cloned, a highly efficient expression system established and a purification protocol determined. The enzyme gives monoclinic crystals in space group $P 2_{1}$, with unit-cell parameters $a=51.5, b=168.7, c=54.9 \AA, \beta=118.8^{\circ}$. A Matthews coefficient $V_{\mathrm{M}}$ of $2.5 \AA^{3} \mathrm{Da}^{-1}$ corresponds to two monomers, each approximately $42 \mathrm{kDa}$ (385 residues), in the asymmetric unit with $50 \%$ solvent content. These crystals are well ordered and data to high resolution have been recorded using synchrotron radiation.

\section{Introduction}

Trypanosoma brucei is a unicellular parasitic protozoan with a complex life cycle involving both insects and mammals. The parasites are transmitted into the bloodstream of mammals by the bite of the tsetse fly, causing African sleeping sickness in humans. No vaccines are available, the drugs in current use have severe side-effects and drug resistance is also rapidly increasing. The enzymes of the mevalonate (MVA) pathway of isoprenoid precursor biosynthesis represent potential drug targets in trypanosomes since active sterol biosynthesis has been shown to be essential for growth and survival in T. cruzi (Urbina, 1997, 2002). For example, sterol-synthesis inhibitors such as terbinafine (an inhibitor of squalene epoxidase, downstream of the MVA pathway) have been shown to retard growth and lead to parasite death (Buckner et al., 2001) and inhibitors of T. cruzi HMGCoA reductase (a central enzyme of the MVA pathway) have been shown to potentiate the anti-proliferative effects of terbinafine (Urbina et al., 1993). This would suggest that inhibition of the MVA pathway offers hope for future drug development against trypanosomatid parasites. Furthermore, several MVA-pathway enzymes, including MDD, have been shown to be essential in yeast (Oulmouden \& Karst, 1990; Tsay \& Robinson, 1991; Bergès et al., 1997) which, like trypanosomatids, is dependent on the MVA pathway for isoprenoid precursor biosynthesis.

Isoprenoids, which represent one of the largest families of compounds in nature, contribute to many important and diverse biochemical roles: for example, quinones participate in electrontransport processes, sterols such as cholesterol or ergosterol are components of membrane structures, dolichol is required for glycoprotein synthesis and isoprenyl moieties are involved in membrane anchorage and also signalling processes (Sacchettini \& Poulter, 1997). The essential building block for all isoprenoids is the five-carbon isopentenyl diphosphate (IPP). Two pathways lead to IPP biosynthesis, those being either the mevalonate (MVA) pathway or the nonmevalonate (also called the 1-deoxy-D-xylulose-5-phosphate or DOXP) pathway (Eisenreich et al., 1998; Rohmer, 1999). Trypanosomes, as is the case for all eukaryotes, archaebacteria and a few eubacteria, use the MVA pathway for IPP biosynthesis. This biosynthetic pathway provides new potential drug targets for exploitation in the search for improved therapies against parasitic 
diseases caused by trypanosomatid infection and it is important to study the enzyme components further.

Mevalonate diphosphate decarboxylase (MDD) catalyses the last step in the pathway, using mevalonate diphosphate in an ATPdependent decarboxylation step to make IPP. MDD is a GHMP kinase superfamily member, so-called because the family originally included galacto-, homoserine, mevalonate and phosphomevalonate kinases. There is interest in this family from a structural perspective since members all share the characteristic $\alpha / \beta$-fold of the GHMP kinase family and similar sequence homology (in the range $10-20 \%$ identity), yet they catalyze different reactions and utilize very different substrates. Only three ternary complex structures are available from this superfamily, including homoserine kinase (Krishna et al., 2001), galactokinase (Hartley et al., 2004) and 4-diphosphocytidyl-2C-methyl-D-erythritol kinase (Miallau et al., 2003), and thus there is limited detailed structural information as to how individual members achieve substrate specificity. Only a single structure of MDD, that of the Saccharomyces cerevisiae enzyme, is available to date and this is in the apo form (Bonanno et al., 2001). Detailed kinetic and mutagenesis studies of this enzyme have suggested a possible mechanism (Dhe-Paganon et al., 1994) and which residues might contribute to substrate binding (Krepkiy \& Miziorko, 2004, 2005), but ligand-complex structures remain essential to achieve a detailed understanding of the catalytic mechanism and ligand specificity of MDD necessary to support a programme of structure-based inhibitor design.

Here, we describe the cloning of the putative mevalonate diphosphate decarboxylase gene from $T$. brucei, the construction of a highly efficient protein-expression system and purification of the recombinant enzyme (of approximate molecular weight $42.4 \mathrm{kDa}, 385$ residues), along with crystallization and preliminary diffraction experiments.

\section{Methods and results}

\subsection{Sample preparation and crystallization}

This project was initiated prior to the annotation of the MDD sequence in the online sequencing database (GeneDB) of the Wellcome Trust Sanger Institute Pathogen Sequencing Unit (http:// www.genedb.org). Similarity matches to all genomic fragments from a preliminary assembly of the T. brucei genome were determined by BLAST search (Altschul et al., 1990) against the SWISS-PROT database. Text mining of the annotation for these sequence matches

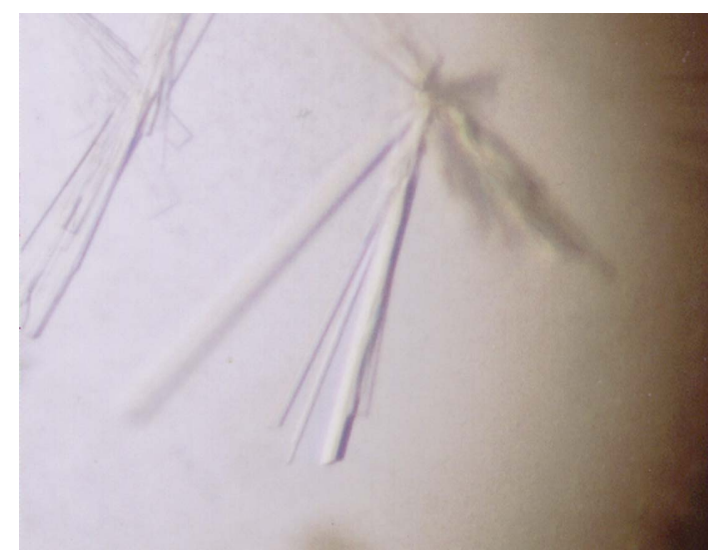

Figure 1

Monoclinic crystals of the putative $T$. brucei MDD. These samples attain a maximum size of $0.2 \times 0.05 \times 0.05 \mathrm{~mm}$. identified a candidate gene sequence for $T$. brucei MDD in contig tryp10.0.000024_21. The predicted gene was amplified from genomic DNA in a PCR reaction using the primers $5^{\prime}$-CATATGTCCGATTCCGATCAGTGTCAGCG- ${ }^{\prime}$ and $5^{\prime}$-GGATCCTCATACCCGAGGTGGAATG-3', containing restriction-enzyme sites for NdeI and BamHI, respectively (bold). The resultant PCR product was cloned into the pCR-BluntII-TOPO shuttle vector using the Zero Blunt TOPO PCR cloning kit (Invitrogen). The vector was cut with NdeI and BamHI to excise the open reading frame and ligated into the pET15b expression vector (Novagen) for expression with an $\mathrm{N}$-terminal hexahistidine tag for ease of purification by metal-ion affinity chromatography. The resulting construct was sequenced to confirm the integrity of the product and then heat-shock transformed into Escherichia coli JM109 cells (Stratagene) for plasmid amplification before transformation into BL21 (DE3) cells (Stratagene) for protein expression. Clones containing the desired plasmid were selected on Luria-Bertani agar plates containing $50 \mu \mathrm{g} \mathrm{ml}^{-1}$ ampicillin (LB/amp). Bacterial cultures were grown in $11 \mathrm{LB} / \mathrm{amp}$ media at $310 \mathrm{~K}$ until a cell density of $\mathrm{OD}_{600}=0.5-0.7$ was achieved; protein expression was then induced overnight at $293 \mathrm{~K}$ with $0.4 \mathrm{mM}$ isopropyl- $\beta$-D-thiogalactopyranoside. Cells were harvested by centrifugation $(3000 \mathrm{~g}, 20 \mathrm{~min}, 277 \mathrm{~K})$, resuspended in $20 \mathrm{~m} M$ Tris$\mathrm{HCl} \mathrm{pH} \mathrm{7.5,} 500 \mathrm{~m} M \mathrm{NaCl}, 5 \mathrm{~m} M$ imidazole and $5 \mathrm{~m} M$ benzamidine and then incubated on ice for $30 \mathrm{~min}$ with lysozyme and DNaseI. Cells were broken by one pass through a French press and the insoluble cell debris removed by centrifugation $(31000 \mathrm{~g}, 30 \mathrm{~min}$, $277 \mathrm{~K})$. The supernatant was filtered and passed over a $5 \mathrm{ml}$ metalchelating column (Hi-Trap; Amersham Biosciences), which had previously been charged with $\mathrm{Ni}^{2+}(100 \mathrm{~m} M$ nickel chloride) and washed with $25 \mathrm{~m} M$ HEPES pH 6.8 containing $50 \mathrm{~m} M \mathrm{NaCl}$ and $5 \mathrm{~m} M$ imidazole. The protein was eluted by running a gradient from $5 \mathrm{~m} M$ to $1 M$ imidazole in $25 \mathrm{~m} M$ HEPES pH 6.8 containing $50 \mathrm{~m} M$ $\mathrm{NaCl}$ (buffer $A$ ); fractions containing MDD were then pooled and dialyzed against buffer $A$. The hexahistidine tag was removed from the protein following incubation at room temperature for $20 \mathrm{~h}$ with 10 units of thrombin (Amersham Biosciences) per milligram of protein. The protein solution was filtered and passed through a second metalchelating column, equilibrated as before and washed with buffer $A$ to remove any uncut protein and tag fragments. After further dialysis against buffer $A$, the protein was loaded onto a $5 \mathrm{ml}$ cation-exchange column (SP Sepharose HP; Amersham Biosciences) previously equilibrated with buffer $A$ for ion exchange. A salt gradient from $50 \mathrm{~m} M$ to $1 M \mathrm{NaCl}$ in $25 \mathrm{~m} M$ HEPES pH 6.8 was applied and MDD passed straight through while contaminants remained on the column. The purity of the MDD sample was assessed by SDS-PAGE and matrix-assisted laser desorption time-of-flight (MALDI-TOF) mass spectrometry. The theoretical weight of the protein is approximately $42.4 \mathrm{kDa}$ and that determined by mass spectrometry was within $50 \mathrm{Da}$. The protein was extensively dialyzed in buffer $A$, concentrated to $25 \mathrm{mg} \mathrm{ml}^{-1}$ and then stored at $277 \mathrm{~K}$. The typical yield of purified protein using this protocol was over $60 \mathrm{mg}$ per litre of bacterial cell culture.

\subsection{Crystallization}

Crystallization trials were initiated using commercially available screens. MDD at $15 \mathrm{mg} \mathrm{ml}^{-1}$ was incubated for at least $2 \mathrm{~h}$ on ice with $2 \mathrm{~m} M$ adenosine $5^{\prime}-(\beta$ - $\gamma$-imido)triphosphate (AMPPNP) and $2 \mathrm{~m} M$ $\mathrm{MgCl}_{2}$. Sitting drops made up of $1 \mu \mathrm{l}$ protein and $1 \mu \mathrm{l}$ reservoir were set up for vapour diffusion against $70 \mu \mathrm{l}$ reservoir solution at $293 \mathrm{~K}$ using 96-well Greiner CrystalQuick plates. An initial hit was obtained in $30 \%$ PEG 8000, $200 \mathrm{~m} M$ ammonium sulfate and $100 \mathrm{~m} M$ sodium 
acetate pH 6.5 (Crystal Screen 1; Hampton Research), which produced small needles overnight. These conditions were optimized in hanging-drop vapour-diffusion experiments by changing the protein solution:reservoir ratio and protein concentration to $3 \mu \mathrm{l}$ protein at $6 \mathrm{mg} \mathrm{ml}^{-1}$ plus $2 \mu \mathrm{l}$ reservoir, with the reservoir ultimately consisting of $22 \%$ PEG $8000,180 \mathrm{mM}$ ammonium sulfate and $100 \mathrm{~m} M$ sodium cacodylate $\mathrm{pH} 6.0$ (Fig. 1). These crystals diffracted to medium resolution $(\sim 2.3 \AA)$ when used on an in-house X-ray source (Fig. 2).

\subsection{X-ray diffraction}

A suitable cryoprotectant was found by screening glycerol, ethylene glycol and 2-methyl-2,4-pentanediol. Finally, MDD crystals were soaked in $15 \%$ glycerol/mother liquor for about $10 \mathrm{~s}$, without any signs of mechanical damage, before being flash-cooled to $103 \mathrm{~K}$ in a stream of nitrogen gas. Crystals were tested and stored in liquid nitrogen and then transported to the European Synchrotron Radiation Facility (ESRF) in Grenoble, France for high-resolution data collection.

Data were measured on beamline ID14-EH4 and autoindexed and processed with MOSFLM (Leslie, 1992), SCALA (Evans, 1997) and the $C C P 4$ suite of programs (Collaborative Computational Project, Number 4, 1994). The crystals were monoclinic, with unit-cell parameters $a=51.5, b=168.7, c=54.9 \AA, \beta=118.8^{\circ}$. Collection of $300^{\circ}$ of data gave a highly redundant data set of good completeness with an effective resolution near $2.0 \AA$ (Table 1). Examination of systematic absences indicates space group $P 2_{1}$. On the basis of gel-filtration and analytical centrifugation experiments, the $T$. brucei MDD appears predominantly as a monomer, which has a molecular weight of approximately $42.4 \mathrm{kDa}$ and consists of 385 amino acids, with traces of a dimer present in solution (unpublished work). A Matthews coefficient $V_{\mathrm{M}}$ (Matthews, 1968) of $2.5 \AA^{3} \mathrm{Da}^{-1}$ correlates to two

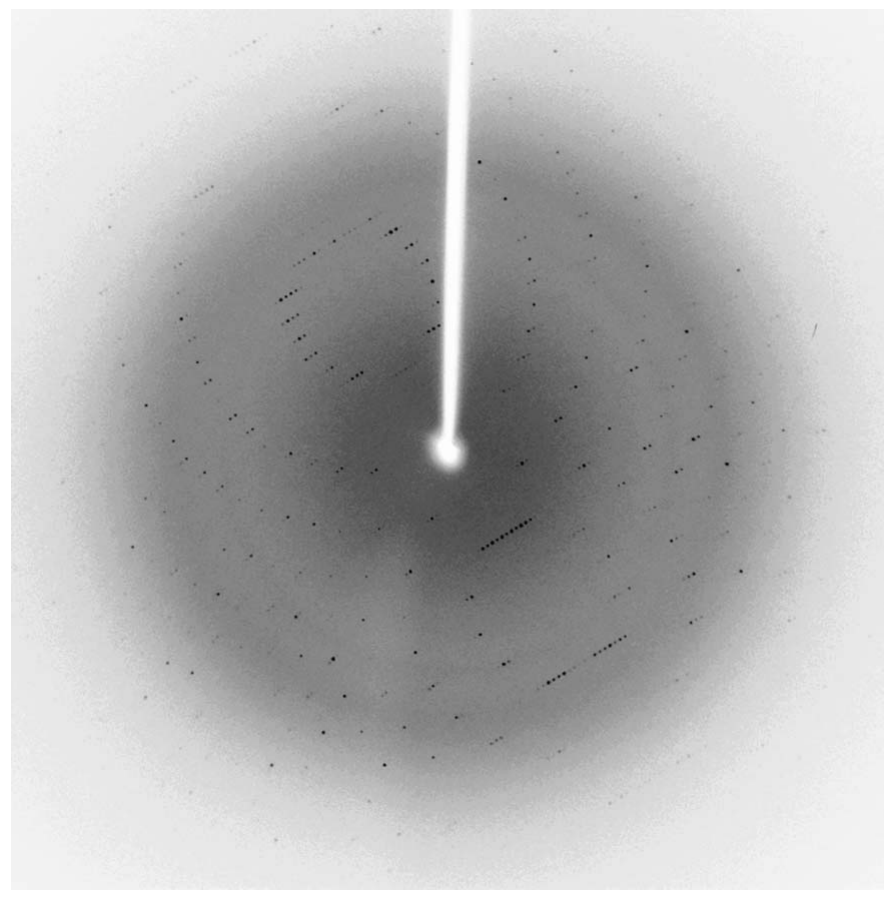

Figure 2

An example of the diffraction obtained in-house using a Rigaku Micromax 007 rotating anode $(\mathrm{Cu} K \alpha, \lambda=1.5418 \AA)$ and R-AXIS IV ${ }^{++}$image-plate detector. The crystal-to-detector distance is $200 \mathrm{~mm}$, the oscillation angle is $0.5^{\circ}$ and the exposure time is $15 \mathrm{~min}$, with X-rays produced at settings of $40 \mathrm{kV}$ and $18 \mathrm{~mA}$.
Table 1

Summary of crystallographic data.

Values in parentheses refer to the highest resolution bin $1.97-1.8 \AA$.

\begin{tabular}{ll}
\hline Resolution range $(\AA)$ & $25-1.8$ \\
Wavelength $(\AA)$ & 0.939 \\
Unit-cell parameters & \\
$\quad a(\AA)$ & 51.5 \\
$b(\AA)$ & 168.7 \\
$c(\AA)$ & 54.9 \\
$\quad \beta\left({ }^{\circ}\right)$ & 118.8 \\
Space group & $P 2_{1}$ \\
No. of measurements & 454564 \\
No. of unique reflections & 73954 \\
Redundancy & 6.1 \\
Completeness $(\%)$ & 99.0 \\
$\langle I / \sigma(I)\rangle$ & $4.3(1.2)$ \\
$R_{\text {sym }}(\%)$ & $10.6(65.8)$ \\
Wilson $B\left(\AA^{2}\right)$ & 24 \\
\hline
\end{tabular}

monomers in the asymmetric unit with $50 \%$ solvent content. A selfrotation function was calculated using MOLREP (Vagin \& Teplyakov, 1997; not shown) and confirmed the presence of a noncrystallographic twofold axis of symmetry $(\psi=66.3, \varphi=28.4$, $\kappa=180.0^{\circ}$ ) with a peak height of approximately $25 \%$ of that observed for the crystallographic peak.

These crystals are suitable for high-resolution crystallographic analyses and, since there is an appropriate model available (yeast MDD; Bonanno et al., 2001), work is under way to solve the structure by the application of molecular-replacement methods. Structure determination will then facilitate the detailed study of the structure and reactivity of MDD in trypanosomatid protozoans.

This work was funded by the Wellcome Trust and BBSRC. We thank Mr Deuan Jones for kindly providing T. brucei brucei (strain 427) genomic DNA, the ESRF for access and Lauris Kemp, Magnus Alphey, Charles Bond and Terence Smith for many helpful discussions.

\section{References}

Altschul, S. F., Gish, W., Miller, W., Myers, E. W. \& Lipman, D. J. (1990). J. Mol. Biol. 215, 403-410.

Bergès, T., Guyonnet, D. \& Karst, F. (1997). J. Bacteriol. 179, 4664-4670.

Bonanno, J. B., Edo, C., Eswar, N., Pieper, U., Romanowski, M. J., Ilyin, V., Gerchman, S. E., Kycia, H., Studier, F. W., Sali, A. \& Burley, S. K. (2001). Proc. Natl Acad. Sci. USA, 98, 12896-12901.

Buckner, F. S., Griffin, J. H., Wilson, A. J. \& Van Voorhis, W. C. (2001). Antimicrob. Agents Chemother. 45, 1210-1215.

Collaborative Computational Project, Number 4 (1994). Acta Cryst. D50, 760 763.

Dhe-Paganon, S., Magrath, J. \& Abeles, R. H. (1994). Biochemistry, 33, 1335513362.

Eisenreich, W., Schwarz, M., Cartayrade, A., Arigoni, D., Zenk, M. H. \& Bacher, A. (1998). Chem. Biol. 5, R221-R223.

Evans, P. R. (1997). Proceedings of the CCP4 Study Weekend. Recent Advances in Phasing, edited by K. S. Wilson, G. Davies, A. W. Ashton \& S. Bailey, pp. 97-102. Warrington: Daresbury Laboratory.

Hartley, A., Glynn, S. E., Barynin, V., Baker, P. J., Sedelnikova, S. E., Verhees, C., De Geus, D., Van Der Oost, J., Timson, D. J., Reece, R. J. \& Rice, D. W. (2004). J. Mol. Biol. 337, 387-398.

Krepkiy, D. \& Miziorko, H. M. (2004). Protein Sci. 13, 1875-1881.

Krepkiy, D. \& Miziorko, H. M. (2005). Biochemistry, 44, 2671-2677.

Krishna, S. S., Zhou, T., Daugherty, M., Osterman, A. \& Zhang, H. (2001). Biochemistry, 40, 10810-10818.

Leslie, A. G. W. (1992). Jnt CCP4/ESF-EACBM Newsl. Protein Crystallogr. 26.

Matthews, B. W. (1968). J. Mol. Biol. 33, 491-497.

Miallau, L., Alphey, M. S., Kemp, L. E., Leonard, G. A., McSweeney, S. M., Hecht, S., Bacher, A., Eisenreich, W., Rohdich, F. \& Hunter, W. N. (2003). Proc. Natl Acad. Sci. USA, 100, 9173-9178. 


\section{crystallization communications}

Oulmouden, A. \& Karst, F. (1990). Gene, 88, 253-257.

Rohmer, M. (1999). Nat. Prod. Rep. 16, 565-574.

Sacchettini, J. C. \& Poulter, C. D. (1997). Science, 277, 1788-1789.

Tsay, Y. Y. H. \& Robinson, G. W. (1991). Mol. Cell Biol. 11, 620-631.

Urbina, J. A. (1997). Parasitology, 114, S91-S99.
Urbina, J. A. (2002). Curr. Pharm. Des. 8, 287-295.

Urbina, J. A., Lazardi, K., Marchan, E., Visbal, G., Aguirre, T., Piras, M. M. \& Piras, R. (1993). Antimicrob. Agents Chemother. 37, 580-591.

Vagin, A. A. \& Teplyakov, A. (1997). J. Appl. Cryst. 30, 1022 1025 